University of Wollongong

Research Online

Faculty of Social Sciences - Papers (Archive) Faculty of Arts, Social Sciences \& Humanities

2006

The effects of training in behaviour modification strategies on stress, burnout, and therapeutic attitudes in frontline inpatient mental health nurses

Hamish J. McLeod

University of Wollongong, hamish@uow.edu.au

Lisa Densley

University of Wollongong

Kate Chapman

Avon \& Wiltshire Mental Health NHS Trust

Follow this and additional works at: https://ro.uow.edu.au/sspapers

Part of the Education Commons, and the Social and Behavioral Sciences Commons

Research Online is the open access institutional repository for the University of Wollongong. For further information contact the UOW Library: research-pubs@uow.edu.au 


\title{
The effects of training in behaviour modification strategies on stress, burnout, and therapeutic attitudes in frontline inpatient mental health nurses
}

\begin{abstract}
Psychiatric nursing is a stressful profession associated with high levels of burnout. Previous research has demonstrated that burnout in psychiatric nurses can be reduced via training that improves behaviour modification skills. However, the minimum amount of training required to demonstrate a beneficial effect is unclear. We evaluated the impact of a 4-day behaviour modification training program on stress, burnout, and therapeutic attitudes in nurses who were in frequent daily inpatient contact with patients with severe mental illnesses. Nurses working in the same wards served as a control group. Training improved therapeutic attitudes but did not alter self ratings of job-stress and burnout. The need for multi-pronged approaches to the prevention and treatment of burnout in psychiatric nurses is discussed.
\end{abstract}

\section{Keywords}

effects, attitudes, inpatient, mental, nurses, stress, modification, burnout, training, therapeutic, frontline, health, strategies, behaviour

\section{Disciplines \\ Education | Social and Behavioral Sciences}

\section{Publication Details}

McLeod, H. J., Densley, L. \& Chapman, K. (2006). The effects of training in behaviour modification strategies on stress, burnout, and therapeutic attitudes in frontline inpatient mental health nurses. The Australian Journal of Rehabilitation Counselling, 12 (1), 1-10. 


\title{
The Effects of Training in Behaviour Modification Strategies on Stress, Burnout, and Therapeutic Attitudes in Frontline Inpatient Mental Health Nurses
}

\author{
Hamish J. McLeod, \\ University of Wollongong, Australia \\ Lisa Densley \\ University of Wollongong, Australia \\ Kate Chapman \\ Early Psychosis Service, Avon and Wiltshire Mental Health Partnership NHS Trust, \\ United Kingdom
}

\begin{abstract}
Dsychiatric nursing is a stressful profession associated with high levels of burnout. Previous research has demonstrated that burnout in psychiatric nurses can be reduced via training that improves behaviour modification skills. However, the minimum amount of training required to demonstrate a beneficial effect is unclear. We evaluated the impact of a 4-day behaviour modification training program on stress, burnout, and therapeutic attitudes in nurses who were in frequent daily inpatient contact with patients with severe mental illnesses. Nurses working in the same wards served as a control group. Training improved therapeutic attitudes but did not alter self ratings of job-stress and burnout. The need for multi-pronged approaches to the prevention and treatment of burnout in psychiatric nurses is discussed.
\end{abstract}

Burnout resulting from occupational stress has potentially serious implications for psychiatric nurses and their employers (Fagin, Brown, Bartlett, Leary, \& Carson, 1995; Melchior, Bours, Schmitz, \& Wittich, 1997; Sullivan, 1993). The burnout syndrome is marked by exhaustion, cynicism and detachment from work roles, and a sense of ineffectiveness and low accomplishment (Maslach, Schaufeli, \& Leiter, 2001). Some of the consequences include impaired job performance, low morale, high job turnover, absenteeism, low work satisfaction, and reduced organisational commitment (Melchior et al., 1997). Broader effects include problems with recruitment and retention of psychiatric nursing staff (Butterworth, Carson, Jeacock, White, \& Clements, 1999). Also, work-related stress and burnout may contribute to the development of anxiety and depressive disorders (Weinberg \& Creed, 2000).

Author for correspondence: Hamish J. McLeod, School of Psychology, University of Wollongong, Northfields Avenue, NSW 2522, Australia. Email: hamish@uow.edu.au 
The development of burnout is linked to sources of job stress such as interacting with violent patients, a perceived lack of support from supervisors and co-workers, and time pressure (Maslach et al., 2001). Melchior et al. (1997) conducted a metaanalysis of studies examining the correlates of burnout in psychiatric nurses and found that job satisfaction, staff support, and organisational commitment were protective factors, while role conflict was an exacerbating factor. They also concluded that unrealistic expectations about the patient's potential for rehabilitation along with frequent aversive interactions with aggressive or suicidal patients elevate the likelihood of developing burnout. This is consistent with the notion that unrealistic attitudes and expectations relating to job performance may lead to the expending of energy with insufficient perceived return and the subsequent development of exhaustion and cynicism (Maslach et al., 2001). One suggested response to this problem is the development of training and supervision interventions that improve nurses' skills (Novak \& Chapel, 1994). This may then lead to improved job related self-efficacy and increase feelings of personal accomplishment from work (Farrington, 1997).

\section{Reducing Nursing Staff Burnout Through Skills Training}

One of the responses to the problem of burnout in frontline clinical nursing staff has been to address skills deficits that may undermine role performance and lead to aversive and unrewarding interactions with patients (Ewers, Bradshaw, McGovern, \& Ewers, 2002). Indirect support for this strategy comes from the finding that younger nurses are at greater risk of burnout, presumably because they are at an early stage of developing and consolidating their clinical skills (Melchior et al., 1997). Several studies using different types of training intervention have examined the effects of training on burnout. Corrigan, McCracken, Edwards, Kommana, and Simpatico (1997) proposed that frontline nursing staff would benefit from training in the application of behaviour modification techniques and delivered interactive 90 -minute training sessions monthly for 8 months. This 12 -hour intervention provided the trainees with strategies for modifying specific problematic patient behaviours and sought to instil more optimistic attitudes toward the use of behaviour modification. Their rationale was that despite empirical evidence in support of the use of behaviour modification techniques in psychiatric rehabilitation (e.g., McMongle \& Sultana, 2006) these strategies are often neglected because of negative attitudes held by clinical staff. Staff who received the training displayed reduced negative attitudes toward behavioural interventions and perceived fewer barriers to their implementation. They also demonstrated a reduction in the emotional exhaustion dimension of burnout compared to their colleagues who did not participate in the training.

Similar findings are reported by Ewers et al. (2002) who delivered a 20-day training program designed to improve knowledge of psychosocial interventions. The participants were forensic psychiatric nurses who were in direct patient contact for more than 35 hours per week. Participation in the training was associated with significant improvements in knowledge about psychosocial interventions, less dysfunctional attitudes toward problematic patient behaviours, and reduced burnout as measured by the Maslach Burnout Inventory (Maslach, Jackson, \& Leiter, 1996). They proposed that reductions in burnout were related to both the development of 
new knowledge and skills as well as the modification of dysfunctional attitudes toward patients.

\section{How Training Might Help Reduce Burnout}

One interpretation of these results is that training reduces burnout via at least two routes: (1) the development of new skills, and (2) the modification of unhelpful attitudes. Skill development may lead to more positive clinical interactions, improved therapeutic outcomes and subsequently greater job satisfaction, higher work related self-efficacy and lower burnout. Milne (1984) has demonstrated that the skill acquisition effects of training in behaviour modification techniques can be maintained up to one year after the completion of a relatively brief training course.

However, even if the requisite skills have been acquired, the implementation of behaviour modification techniques by frontline nursing staff will be influenced by attitudes (Corrigan et al., 1998). Influential attitudes include those focused on organisational factors (e.g., the employing institution does not provide sufficient resources to support behavioural interventions, colleagues are unsupportive), philosophical beliefs about the intervention (e.g., behaviour modification is ineffectual or unethical), and attitudes toward the patient and their condition (e.g., the symptoms of schizophrenia are too severe for psychological interventions to be effective; a lack of motivation in patients reflects 'laziness' rather than the negative symptoms of schizophrenia) (McLeod, Deane, \& Hogbin, 2002; Corrigan, Kwartarini, \& Pramana, 1992). Hence, in addition to fostering the development of clinical skills, training may present an opportunity to modify attitudes that have previously contributed to reduced role-engagement and burnout. McLeod et al. (2002) demonstrated that a 3-day training course in the use of cognitive-behavioural therapy techniques for psychosis promoted positive attitude changes in domains such as empathy for patients' experience, feelings of legitimacy to intervene psychologically, and adequacy regarding clinical skills. Changes were also observed in work-related self esteem and work satisfaction, both of which are protective against burnout. However, the 77 participants were from various professional backgrounds and had varying amounts of direct daily patient contact. Also, burnout was not explicitly measured and there was no comparison with an untrained control group.

\section{The Current Study}

There are suggestions that relatively brief training can produce changes in attitudes that are related to burnout (McLeod et al., 2002) and there is evidence that training delivered over periods of several months improves the implementation of psychological interventions and ameliorate self-rated burnout (Corrigan et al., 1997; Ewers et al., 2002). However, the optimal duration of training and the spacing of trainer input required to yield beneficial effects is unclear. This is relevant because postqualifying training for mental health nurses is often delivered by an external expert who provides concentrated input over a short period of time. The current study investigated this issue by evaluating externally provided training in behaviour modification delivered to psychiatric nurses who were in frequent direct clinical contact with psychiatric inpatients with severe mental illnesses. A central aim was to determine the effects of a 4-day training course on changes in attitudes, burnout, job-related stress, and self-evaluation of skills and competence to use behaviour modification 
techniques. It was predicted that compared to a control group of ward-based colleagues who did not receive the training, the course participants would show improvements in therapeutically positive attitudes toward working with the severely mentally ill and reductions in burnout following training.

\section{Method}

\section{Participants}

Twenty-seven mental health nurses working in psychiatric inpatient units in Australia and the United Kingdom participated. Nineteen of these received the training and eight served as a yoked control group who completed the study measures at the same points in time. Participants voluntarily completed the study measures and participation in the training was not contingent on contributing data. Apart from small amounts of missing data, all participants completed the measures to the level required for investigation of the study aims. There were no significant differences between the experimental and control group on variables on any of the clinical experience and demographic variables (see Table 1).

\section{Design}

A quasi-experimental repeated measures design was used. Participants were selected randomly from all ward-based nursing staff in two inpatient units following a general call for expressions of interest in participating in the training. Participants were randomly allocated to either the behaviour modification training condition or the no training control condition. Measures were taken immediately before and immediately after the training course for all subjects (within one week of the training dates). This was done in an attempt to control for the influence work related events that might have contributed to job stress.

\section{Training Course Structure and Content}

The training program consisted of four 6-hour sessions delivered over a 4-week period to small groups of four or five nurses. The course content included an introduction to behavioural modification concepts such as classical conditioning, operant conditioning, reinforcement, punishment, extinction, shaping and modelling. Participants were taught how to gather baseline data prior to treatment; follow-up

\section{TABLE 1}

Demographic Characteristics and Clinical Experience of Study Participants

\begin{tabular}{lcccc}
\hline & \multicolumn{2}{c}{ Training participants } \\
$(n=19)$ & Mean & $S D$ & \multicolumn{2}{c}{$\begin{array}{c}\text { Control } \\
(n=8)\end{array}$} \\
\hline Age (years) & 47.13 & 9.21 & 45.29 & 8.26 \\
Age range (years) & $25-59$ & & $31-54$ & \\
Years of experience & 22.06 & 8.45 & 16.37 & 8.41 \\
Male & 13 & 3 & & \\
Female & 6 & 5 & & \\
\hline
\end{tabular}


data after treatment; how to teach new behaviours using chaining; the use of extinction; and the modification of unwanted behaviours via differential reinforcement of incompatible behaviour. In between training sessions the participants were required to complete homework tasks involving the application of behaviour modification strategies to a patient under their care. Videotapes were used to develop skills in behaviour monitoring and feedback was given on the development of participants skills as the course progressed. The training was delivered by either a doctoral level clinical psychologist or a master's level trainee in clinical psychology and the course content development and delivery of training was supervised by a senior clinical psychologist.

\section{Measures and Procedure}

All of the study measures were self-report questionnaires that were completed immediately prior to each training event and immediately after the final session. The control subjects were given the measures at the same time and asked to complete and return them within one week.

Burnout was measured with The Maslach Burn-Out Inventory: Third Edition (MBI; Maslach, Jackson \& Leiter, 1996). This 22-item questionnaire is the most commonly used tool for studying burnout in the caring professions (Maslach et al., 2001) and comprises three main subscales: Emotional Exhaustion (EE), Depersonalisation (DP), and Personal Accomplishment (PA). A 7-point Likert scale is used to rate the frequency of positive and negative job-related experiences (from 0 = never to 6 = every day). Sample items include 'I feel used up at the end of the workday' and 'I feel like I am at the end of my rope'. The subscales have demonstrated reliability and validity and the scale is suitable for repeated administration (Lee \& Ashforth, 1993).

In recognition that job-related stress is a potential precursor to burnout but is not synonymous with it, we included the DeVillers Carson Leary Stress Scale, Version 3 (DCL; Carson et al., 1994). The DCL is a 30-item measure of occupational stress designed specifically for use with ward-based psychiatric nurses. The measure has adequate internal (Cronbach Alpha $=0.96$ ) and test retest reliability (Spearman's Rho $=0.91$ ), as well as satisfactory face, content and concurrent validity (Fagin et al., 1996). Sources of potential job stress are listed (e.g., 'having to deal with suicidal patients') and responses are rated on a 5-point Likert scale $(0=$ no stress to 4 = extremely stressed $)$. The DCL yields five subscales that reflect sources of work related stress: Patients Demands, Organisational and Managerial Issues, Staffing, Future Concerns, and Job Satisfaction.

The 28 item version of the General Health Questionnaire (GHQ; Goldberg, 1981) was used to measure general psychological distress and physical health. This widely used self-report measure has excellent psychometric properties and is sensitive to the presence of psychiatric disturbance. Scores above 5 reflect 'psychiatric caseness' suggestive of the presence of an anxiety or depressive disorder (Weinberg $\&$ Creed, 2000).

Attitudes relating to using behavioural interventions in inpatient settings were assessed with the Barriers to Implementation of Behaviour Therapy scale (BIBT; Corrigan et al., 1992). This scale consists of 18 statements about barriers to behavioural innovations with each statement rated on a 6-point scale from $1=$ strongly agree to 6 strongly disagree. Previous factor analysis has confirmed the presence of 
three main subscales: (1) institutional constraints (e.g., 'there is too much staff turnover to consistently carry out behaviour modification'), (2) insufficient collegial support (e.g., 'fellow staff members are not interested in carrying out behaviour modification'), and (3) philosophical opposition (e.g., 'behaviour modification is unethical'). Lower factor scores indicate greater endorsement of the barrier. Additional attitudes were measured with an adapted version of the 34 item scale used by McLeod et al. (2002) in their study of participants in a training program addressing CBT for psychosis skills. Respondents use a 7-point Likert scale $(1=$ strongly disagree to 7 = strongly agree $)$ to rate their agreement with statements such as 'schizophrenia is too severe a condition for behaviour modification to be effective with' and 'at times I am no good at working with people who have schizophrenia'. The majority of the items were derived from existing measures of attitudes toward working with people with substance misuse problems and yields five main subscales: adequacy, legitimacy, motivation, work satisfaction, and work-related self esteem. Other items were adapted from previous work on clinician beliefs about people with schizophrenia. The scale is sensitive to attitude changes following training interventions (see McLeod et al., 2002, for a more detailed description of the scale and its psychometric properties).

\section{Analysis}

All data analyses were completed using the Statistical Package for the Social Sciences (SPSS, 1999) with alpha set at .05. Nonparametric tests were used for group comparisons and repeated measures analysis.

\section{Results}

A crucial prerequisite for testing the study hypotheses is the assumption that the control group's scores would remain unchanged across both assessment time points. This assumption was supported; no significant differences were found for the control group at Time 1 versus Time 2 . Also, the scores for the participants who received training and the control group were not significantly different at baseline, with the exception of the beliefs about schizophrenia subscale from McLeod et al.'s (2002) attitudes measure (Mann-Whitney $U=35.5, Z=-2.16, p<.05$ ). Thus, this subscale was excluded from further analyses. The stability of the control subjects' scores over time and the equivalence of scores at baseline for the control and experimental groups bolsters the interpretation that any observed changes in scores for the trained participants are attributable to the training received.

The effects of training on measures of burnout, job-related and stress, and general distress were assessed by comparing the MBI, DCL, and GHQ scores before and after training (see Table 2). No significant difference was observed for pre- and posttraining scores for any of these measures and so the hypothesis that experiencing the training would directly reduce indices of stress and burnout was not supported.

The second hypothesis was that the participants receiving training would show changes in attitudes relating to working with people with schizophrenia and the use of psychological interventions to alleviate problem behaviours. This hypothesis was broadly supported. The Philosophical Opposition subscale of the BIBT showed a 
TABLE 2

Measures of Job Stress, Burnout, and Psychological Distress Before and After Training

\begin{tabular}{|c|c|c|c|c|c|c|c|c|}
\hline & \multicolumn{4}{|c|}{ Training participants } & \multicolumn{4}{|c|}{ Control } \\
\hline & \multicolumn{2}{|c|}{ Time 1} & \multicolumn{2}{|c|}{ Time 2} & \multicolumn{2}{|c|}{ Time 1} & \multicolumn{2}{|c|}{ Time 2} \\
\hline & Mean & $S D$ & Mean & $S D$ & Mean & $S D$ & Mean & $S D$ \\
\hline \multicolumn{9}{|l|}{$\mathrm{MBI}$} \\
\hline 1. EE & 15.26 & 10.20 & 15.42 & 11.38 & 20.12 & 10.47 & 19.00 & 8.78 \\
\hline 2. $D$ & 5.16 & 4.49 & 5.42 & 5.09 & 7.50 & 6.30 & 6.00 & 6.09 \\
\hline 3. $\mathrm{PA}$ & 34.00 & 8.01 & 33.16 & 8.51 & 35.75 & 7.48 & 30.00 & 7.56 \\
\hline \multicolumn{9}{|l|}{$\mathrm{DCL}$} \\
\hline Total & 52.74 & 19.91 & 49.74 & 20.19 & 57.63 & 20.47 & 57.75 & 18.86 \\
\hline \multicolumn{9}{|l|}{$\mathrm{GHQ}$} \\
\hline Total & 3.05 & 5.03 & 2.61 & 4.58 & 2.37 & 5.01 & 2.37 & 4.78 \\
\hline
\end{tabular}

Notes: $\mathrm{MBI}=$ Maslach Burnout Inventory ( $\mathrm{EE}=$ emotional exhaustion; $\mathrm{D}$ = depersonalisation;

$\mathrm{PA}=$ personal accomplishment).

$D C L=$ de Villiers, Carson, Leary Stress Scale.

$\mathrm{GHQ}=$ General Health Questionnaire.

\section{TABLE 3}

Measures of Attitudes Before and After Training

\begin{tabular}{lccccccccc}
\hline & \multicolumn{3}{c}{ Training participants } & \multicolumn{5}{c}{ Control } \\
\cline { 2 - 11 } & \multicolumn{2}{c}{ Time 1 } & \multicolumn{2}{c}{ Time 2 } & \multicolumn{2}{c}{ Time 1 } & \multicolumn{2}{c}{ Time 2 } \\
\cline { 2 - 11 } & Mean & SD & Mean & SD & Mean & SD & Mean & SD \\
\hline BIBT & & & & & & & & \\
1.Constraints & 14.95 & 3.47 & 15.89 & 4.42 & 14.50 & 3.66 & 13.37 & 3.20 \\
2. Collegial support & 12.95 & 4.40 & 12.37 & 4.49 & 15.25 & 3.91 & 14.00 & 4.84 \\
3. Philosophical* & 12.67 & 3.41 & 13.94 & 2.84 & 11.12 & 1.13 & 11.12 & 0.83 \\
Attitudes & & & & & & & & \\
1. Motivation* & 28.47 & 4.17 & 30.16 & 4.41 & 29.75 & 3.95 & 29.00 & 2.62 \\
2. Work satisfaction & 26.84 & 28.73 & 28.73 & 4.29 & 29.12 & 3.40 & 26.50 & 4.28 \\
3. Self-esteem* & 25.37 & 4.72 & 27.47 & 4.36 & 29.50 & 4.11 & 27.62 & 3.85 \\
4. Adequacy* & 27.79 & 4.68 & 30.12 & 5.56 & 30.88 & 5.84 & 30.87 & 8.32 \\
5. Legitimacy* & 19.16 & 5.47 & 21.21 & 3.52 & 22.62 & 3.38 & 20.87 & 4.05
\end{tabular}

Note: $\mathrm{BIBT}=$ Barriers to Implementation of Behaviour Therapy scale.

${ }^{*}$ Measures that showed significant differences following training.

significant change following training (Wilcoxon Signed Ranks Test, $Z=2.91$, $p<.005$, two-tailed) while the other two subscales were unchanged (see Table 3 ).

Further evidence that training modified staff attitudes was found on participants responses to McLeod et al.'s (2002) measure (see Table 3). The training participants 
showed increases in motivation $(Z=-1.86, p<.05)$, work-related self-esteem $(Z=-2.33, p<.05)$, adequacy $(Z=-2.55, p<.05)$, and legitimacy $(Z=-1.67$, $p<.05)$ following training. No change was observed for self-ratings of work satisfaction.

\section{Discussion}

This study investigated whether a relatively brief and focused training program in the use of behaviour modification techniques would have any beneficial effects on the pernicious problem of burnout amongst psychiatric nurses. In contrast to previous research the results of the current study do not indicate that the training program used here was effective in producing changes in stress, burnout, or psychological distress. However, there was evidence that participating in the training produced changes in attitudes that, if maintained, may lead to behavioural change that may ameliorate or prevent burnout. The conclusion that this attitude change was due to the training is made more secure by the fact that the participants who did not receive training showed no significant differences in attitude measure scores across the two assessment points.

Given that previous authors have reported favourable effects of training in therapeutic skills on burnout (Corrigan et al., 1997; Ewers et al., 2002), some possible explanations for the lack of an effect in the current study is warranted. Two explanations are proposed. First, the training program offered here was delivered in a relatively concentrated timeframe compared to other studies which have delivered training over 6 months or longer. This prolonged training window may be critical for training recipients to practice the skills taught, receive feedback, and have sufficient time for the implementation of the new skills to lead to observable favourable therapeutic outcomes. While efforts were made to get the training participants in the current study to apply their new skills during the training period it is possible that they did not have sufficient opportunity to gain positive therapeutic experiences that were sufficient to improve facets of burnout such as job satisfaction. Although a considerable amount of training in mental health settings is delivered intensively over a brief period of a few days, the current results suggest that this delivery format may not reliably reduce burnout and work related stress.

Another interpretation for cautious consideration is that the observed positive effects of training on therapeutic attitudes actually reflect a small effect on burnout that was insufficient to be detected by changes in the group averages on the MBI. This is supported by prior research that used path analysis of the relationship between BIBT and MBI scores and found burnout leads to negative attitudes about behaviour therapy but not the reverse (Corrigan et al., 1998).

A second explanation for a lack of direct impact of therapeutic skills training on measurable burnout and job related stress is that the cause of these problems is multidimensional (Corrigan et al., 1998; Edwards \& Burnard, 2003). Stress at work is one source of emotional burden but other factors such as personal stressors, insufficient social support, and impaired coping responses may also contribute to ineffectiveness, emotional exhaustion, and reduced satisfaction with life roles (Weinberg \& Creed, 2000). Only modifying an individual's skill in dealing with challenging or aversive patient behaviours may leave other sources of stress unaddressed and consequently little change in burnout will be achieved. This reflects the need for multi-pronged 
interventions that include strategies such as personal stress management training, enhancement of collegial and other social support, and ongoing clinical supervision that include skill development and restorative elements (Butterworth et al., 1999; Edwards \& Burnard, 2003). Finally, it is possible that the relatively small sample size in the present study did not have sufficient power to detect changes in burnout, stress and distress.

\section{Conclusions}

This study partly replicates McLeod et al.'s (2002) finding that training in psychological interventions for serious psychiatric illnesses can foster stronger endorsement of positive attitudes toward working therapeutically with patients in need of rehabilitation. Helping frontline clinical staff to be motivated to use effective psychological therapy techniques may be a critical component in maintaining a positive attitude toward and engagement with work roles. Also, the potential for short and focused training courses to be beneficial is evident from the training recipients observed improvements in self-esteem, appraisals of personal adequacy, and beliefs that it is legitimate to use empirically supported psychological strategies in daily patient care. However, addressing burnout is likely to require a longer period of supervision, monitoring, support and feedback. Individuals experiencing more severe burnout and those at risk of developing burnout will benefit from a multi-pronged approach that addresses factors both within the individual (e.g., self-care skills and therapeutic skills) and their environment (e.g., organisational factors, social support).

\section{Acknowledgements}

The contribution of the research participants is gratefully acknowledged. Jerome Carson is thanked for providing the DCL.

\section{References}

Butterworth, T., Carson, J., Jeacock, J., White, E., \& Clements, A. (1999). Stress, coping, burnout and job satisfaction in British nurses: findings from the clinical supervision evaluation project. Stress Medicine, 15, 27-33.

Carson, J., Leary, J., Bartlett, H., de Villiers, N., Fagin, L., Holloway, F., et al. (1994). Manual for the mental health nursing stress questionnaires. London: The Bethlem and Maudsley NHS Trust.

Corrigan, P.W., Kwartarnini, W.Y., \& Pramana, W. (1992). Staff perception of barriers to behavior therapy at a psychiatric hospital. Behavior Modification, 16, 132-144.

Corrigan, P.W., McCracken, S.G., Edwards, M., Kommana, S., \& Simpatico, T. (1997). Staff training to improve implementation and impact of behavioral rehabilitation programs. Psychiatric Services, 48(10), 1336-1338.

Corrigan, P.W., Williams, O.B., McCracken, S., Kommana, S., Edwards, M., \& Brunner, J. (1998). Staff attitudes that impede the implementation of behavioral treatment programs. Behavior Modification, 22, 548-562.

Edwards, D., \& Burnard, P. (2003). A systematic review of stress and stress management interventions for mental health nurses. Journal of Advanced Nursing, 42, 169-200.

Ewers, P., Bradshaw, T., McGovern, J., \& Ewers, B. (2002). Does training in psychosocial interventions reduce burnout rates in forensic nurses? Journal of Advanced Nursing, 37, 470-476.

Fagin, L., Brown, D., Bartlett, H., Leary, J., \& Carson, J. (1995). The Claybury community psychiatric nurse stress study: Is it more stressful to work in hospital or the community? Journal of Advanced Nursing, 22, 347-356. 
Fagin, L., Carson, J., Leary, J., de Villiers, N., Bartlett, H., O’Malley, P., et al. (1996). Stress, coping and burnout in mental health nurses: Findings from three research studies. International Journal of Social Psychiatry, 42, 102-111.

Farrington, A. (1997). Strategies for reducing stress and burnout in nursing. British Journal of Nursing, 6, 44-49.

Goldberg, D. (1981). Manual for the general health questionnaire. Windsor, United Kingdom: NFERNelson.

Lee, R.T., \& Ashforth, B.E. (1993). A longitudinal study of burnout among supervisors and managers: Comparisons between the Leiter and Maslach (1988) and Golembiewski et al. (1986) models. Organizational Behavior and Human Decision Processes, 54, 369-398.

Maslach, C., Jackson, S.E., \& Lieter, M.P. (1996). Maslach burnout inventory manual (3rd ed.).: Palo Alto, CA: Consulting Psychologists Press.

Maslach, C., Schaufeli, W.B., \& Leiter, M.P. (2001). Job burnout. Annual Review of Psychology, 52, 387-422.

McLeod, H.J., Deane, F.P., \& Hogbin, B. (2002). Changing staff attitudes and empathy for working with people with psychosis. Behavioural and Cognitive Psychotherapy, 30, 459-470.

McMongle, T., \& Sultana, A. (2006). Token economy for schizophrenia. Cochrane Database of Systematic Reviews, 3.

Melchior, M.E.W., Bours, G.J.J.W, Schmitz, P., \& Wittich, Y. (1997). Burnout in psychiatric nursing: a meta-analysis of related variables. Journal of Psychiatric and Mental Health Nursing, 4, 193-201.

Milne, D. (1984). The development and evaluation of a structured learning format introduction to behaviour therapy for psychiatric nurses. British Journal of Clinical Psychology, 23, 175-185.

Novak, M., \& Chapel, N.L. (1994). Nursing assistant burnout and the cognitively impaired elderly. Aging and Human Development, 39, 105-120.

SPSS (1999). Statistical package for the social sciences Base 10 for Windows. Chicago: Author.

Sullivan, P.J. (1993). Occupational stress in psychiatric nursing. Journal of Advanced Nursing, 18, 591-601.

Weinberg, A., \& Creed, F. (2000). Stress and psychiatric disorder in healthcare professionals and hospital staff. The Lancet, 355, 533-537. 